\title{
Photon-Induced Dissociation with a Four-Sector Tandem Mass Spectrometer
}

\author{
Stephen A. Martin* and James A. Hill \\ Department of Chemistry, Massachusetts Institute of Technology, Cambridge, Massachusetts, USA
}

Carter Kittrell

Department of Chemistry, Rice University, Houston, Texas, USA

\section{Klaus Biemann}

Department of Chemistry, Massachusetts Institute of Technology, Cambridge, Massachusetts, USA

\begin{abstract}
The feasibility of using photodissociation of protonated peptide molecules to sequence specific fragment ions with a 193-nm pulsed laser beam in a magnetic deflection tandem mass spectrometer of EBEB configuration was demonstrated. Although the short pulse (15 ns) and low repetition rate $(100 \mathrm{~Hz})$ of the excimer laser permitted the irradiation of only $\sim 0.02 \%$ of the $(\mathrm{M}+\mathrm{H})^{+}$ions exiting MS-1, a photon-induced decomposition spectrum of the heptapeptide angiotensin III $\left(M_{\mathrm{r}} 930.5\right)$ was produced that was practically the same (but with better signal-to-noise ratio) as that generated by collision-activated dissociation at the same low duty cycle. Because of the low and pulsed fragment ion currents, an array detector was used to record the spectra. A dependence between laser power and abundance of fragment ions was observed (increased power increases the relative abundance of ions of low mass). Laser power was varied from 6 to $80 \mathrm{~mJ}$. Formation of fragment ions from a large peptide (melittin, $M_{\mathrm{r}}$ 2844.75) was also observed. The results permit the design of modifications that may increase the fragment ion yield to $10 \%$ or higher, which would make photon-induced decomposition a useful method for magnetic deflection mass spectrometers. (J Am Soc Mass Spectrom 1990, 1, 107-109)
\end{abstract}

$\mathrm{P}$ hotodissociation of ions by pulsed lasers of a wavelength $(\sim 200 \mathrm{~nm})$ that is absorbed by the carbonyl group of the peptide bond was carried out in the past with Fourier transform mass spectrometers $[1,2]$ that operate in the pulsed mode. We have evaluated the feasibility of pulsed UV laser photoninduced dissociation (PID) in continuous-beam mass spectrometers, such as a four-sector tandem instrument (JEOL $\mathrm{HX110/HX110)} \mathrm{[3].} \mathrm{The} \mathrm{major} \mathrm{difficulty} \mathrm{is}$ the low duty factor due to the short pulse width and low frequency of the excimer lasers presently available (e.g., $15 \mathrm{~ns}$ at $100 \mathrm{~Hz}$ ), which cause only a very small fraction of the continuous precursor ion beam (approximately $0.02 \%$, depending on its velocity) actually to be irradiated during passage through the 25-mm-long PID cell (Figure 1). To observe any effect under those conditions, one has to select a peptide that generates a high $(\mathrm{M}+\mathrm{H})^{+}$ion current, prevent the vast excess of ions not to be irradiated from entering the irradiation region, and use an integrating detector. For these reasons, a relatively small peptide (angiotensin III, RVYIHPF, $M_{\mathrm{r}}$ 930.51) was selected; a pair of electrodes were placed in front of the irradiation cell to

*Present address: Genetics Institute, Andover, Massachusetts, USA. Address mprint requests to Klaus Riemann, Department of Chemistry, Massachusetts Institute of Technology, Cambridge, MA 02139, USA. deflect the ion beam most of the time, except when the transmitted ions are actually irradiated by the 15ns pulse; and an integrating array detector was used $[4,5]$. For precursor ions of $m / z \sim 1000$ (such as angiotensin III), the beam is allowed to pass into the absorption region for $2.2 \times 10^{-6} \mathrm{~s}, 100$ times per second, a total of $2.2 \times 10^{-4} \mathrm{~s} / \mathrm{s}$. Although the cell is illuminated for only $\sim 1.5 \times 10^{-6} \mathrm{~s} / \mathrm{s}$, laser defocusing allows irradiation of virtually all ions in the cell, which is just filled by the $2.2-\mu \mathrm{s}$ ion pulse. Thus, $0.02 \%$ of the total ion beam generated in the ion source is irradiated. A Questek (Billerica, MA) R2440 excimer laser, $193 \mathrm{~nm}, 15 \mathrm{~ns}$ pulse width, $100 \mathrm{~Hz}$, and $275 \mathrm{~mJ}$ maximum power, was used in these experiments.

For optimizing the experimental design and evaluating the photodissociation, His-Ser $\left(M_{\mathrm{r}} 242.09\right)$ was chosen because the immonium ion of $\mathrm{His}(\mathrm{m} / \mathrm{z} 110)$ is one of the most abundant fragments in the collisionactivated dissociation (CAD) spectra of peptides containing this amino acid. A very abundant immonium ion was indeed observed for His with a laser power of $6.8 \mathrm{~mJ} /$ pulse. That this ion is formed because of laser irradiation rather than metastable decomposition was demonstrated by the complete disappearance of the $m / z 110$ peak when the laser beam was turned off and all other experimental parameters were kept identical.

Angiotensin III [RVYIHPF, $(\mathrm{M}+\mathrm{H})^{+}$931.51] pro- 


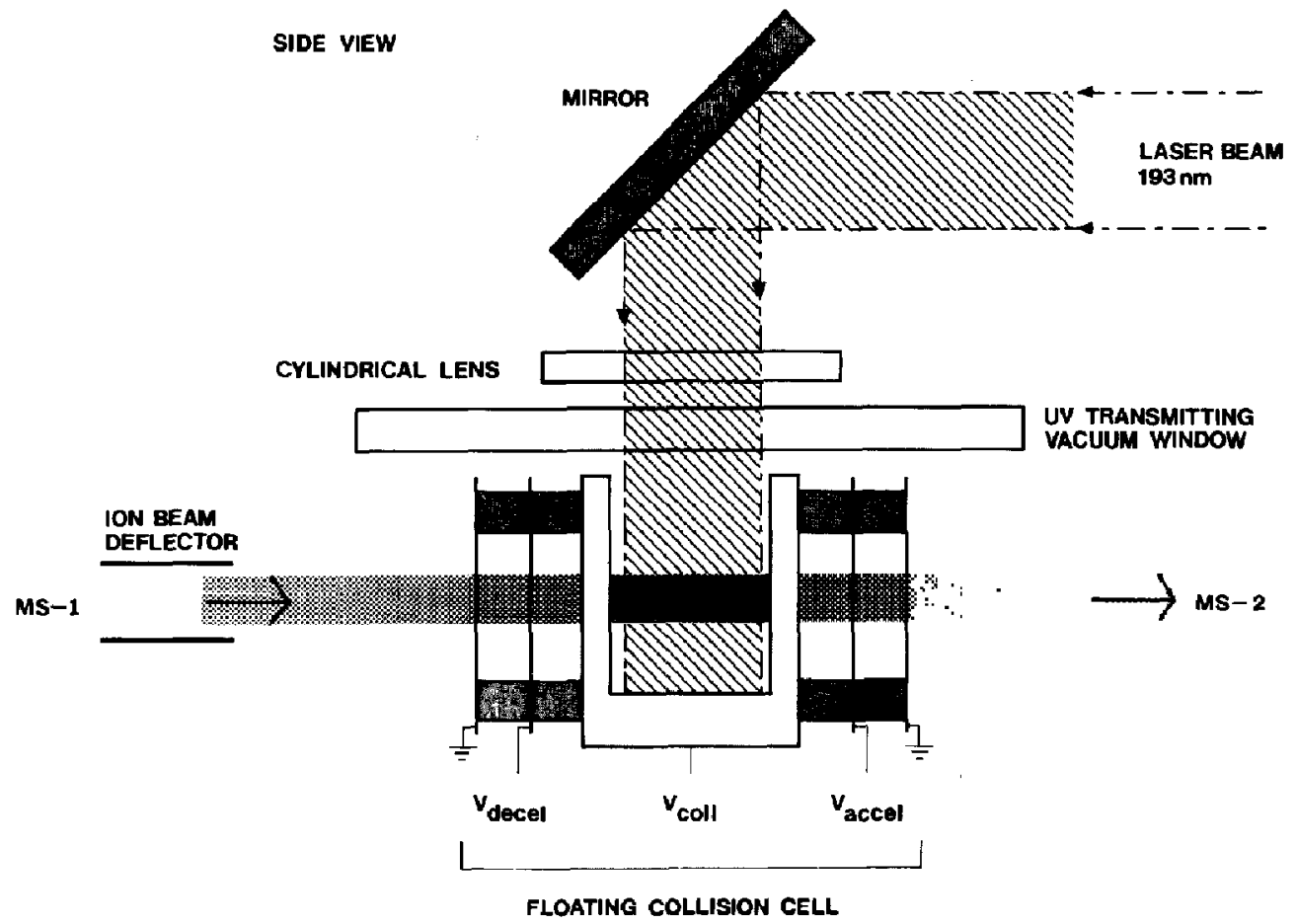

Figure 1. Side view of the laser absorption cell. Ions that exit MS-1 are pulsed by deenergizing the deflector into the absorption region at the same rate as the repetition rate of the laser. Laser beam dimensions are $25 \mathrm{~mm} \times 0.5 \mathrm{~mm}$. Precursor ions are decelerated to $5 \mathrm{keV}$, and the products of the irradiation are then reaccelerated to nominal $10 \mathrm{keV}$ and analyzed in MS- 2 .

duced a PID tandem mass spectrometry (MS/MS) spectrum (Figure 2a) that exhibited all the fragments that are observed in a conventional gas collision experiment acquired at the same low duty cycle (Figure $2 b$ ). The overall sensitivity of the PID spectrum appeared to be significantly higher (as demonstrated by the higher signal-to-noise $(\mathrm{S} / \mathrm{N})$ ratio of Figure 2a) than that of a CAD spectrum (Figure 2b). The CAD spectrum in Figure $2 b$ was acquired under the same experimental conditions as the spectrum in Figure $2 \mathrm{a}$ (including the beam deflection periods), with the exception that the laser was turned off and helium was admitted to the collision cell at a pressure that attenuated the precursor to $30 \%$ of its original intensity. In the absence of either the laser beam or a collision gas, no signals below $\mathrm{m} / \mathrm{z} 890$ were observed (Figure $2 \mathrm{c}$ ), indicating that the fragment ions in Figure $2 a$ and $b$ are indeed produced by the PID and CAD processes, respectively.

The effect of laser power on the type and relative abundance of fragment ions was also investigated with angiotensin III as the test sample at levels ranging from 6 to $80 \mathrm{~mJ} / \mathrm{pulse}$ (measured at the absorption cell). It was found that the relative abundance of the product ions is dependent on the laser power: increasing low mass and decreasing high mass ion abundance with increasing laser power and vice versa. It should be possible to exploit this feature in combination with an array detector by varying the laser power as a function of the consecutive mass windows recorded by the array detector. Thus optimum sequence ion abundances could be obtained over the entire PID spectrum.

For a preliminary evaluation of the efficiency of photodissociation of $(\mathrm{M}+\mathrm{H})^{+}$ions of much higher mass, the $(\mathrm{M}+\mathrm{H})^{+}$ion of melittin $\left(M_{\mathrm{r}} 2844.75\right)$ was irradiated in an experiment. Whereas the precursor ion current $(m / z 2845.8)$ was much too low to observe a complete spectrum, the use of long integrations of the signal $(240 \times 0.6 \mathrm{~s})$ on the array detector demonstrated the formation of immonium ions of lysine $(\mathrm{m} / \mathrm{z} 84)$ and leucine or isoleucine ( $m / z 86)$ upon irradiation at $80 \mathrm{~mJ}$ laser power.

\section{Conclusions}

These experiments indicated the approximate laser power required for dissociation and the experimental parameters necessary to acquire PID mass spectra. The results indicate that PID is feasible in a magnetic deflection tandem mass spectrometer and suggest that the types of fragment ions are the same as those observed in high-energy (keV) CAD experiments [6] and that the extent of fragmentation depends on the laser power. An advantage of PID is that the type of fragment ions observed is independent of the kinetic energy of the precursor ions because the internal energy is due to interaction with high-energy photons and does not arise 

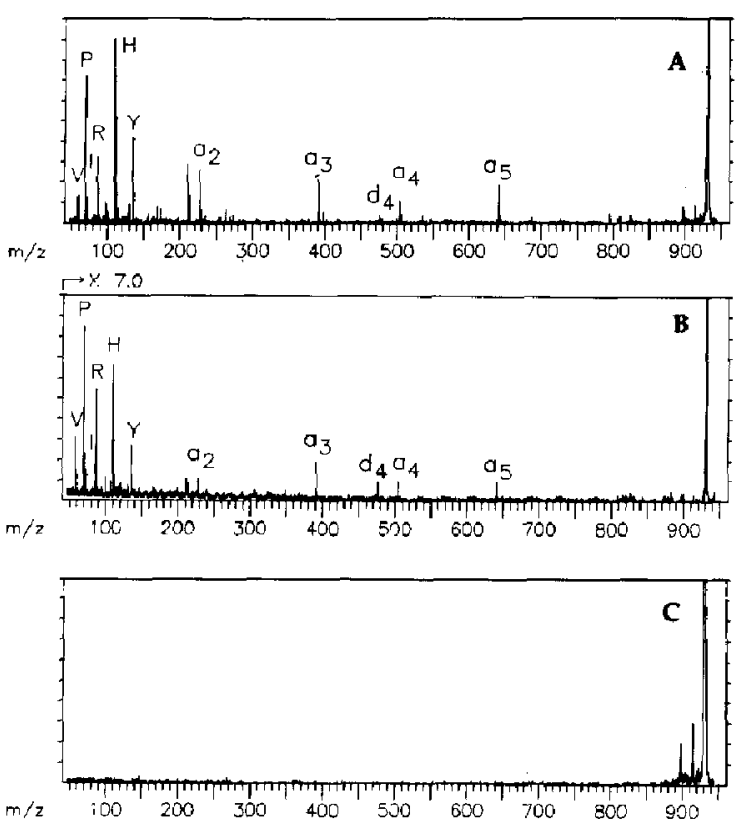

Figure 2. Comparison of the efficiency of PID versus CAD MS/MS for angiotensin III (Arg-Val-Tyr-Ile-His-Pro-Phe, $M_{r}$ 930.5). In (a)-(c) the precursor ions are pulsed into the collision cell at $100 \mathrm{~Hz}$ with a pulse width of $2.2 \mu \mathrm{s}$. (a) Laser on at 6.8 $\mathrm{mJ} /$ pulse; (b) helium rollision gas instead of laser beam; (c) laser off, no gas. All spectra were recorded in $\mathbf{4 8}$ steps (ten integrations of $0.6 \mathrm{~s}$ each) with an array detector. For the peak notations, see ref 6 .

from collision with a neutral atom. At the same time, refocusing the fragment ion beam onto the entrance slit of the second mass spectrometer may be facilitaled by the absence of gas scattering in the MS/MS interface.

To increase the fragmentation efficiency to a point where PID of complex molecules becomes practical in a four-sector tandem mass spectrometer, we plan the fol- lowing modifications: (1) Increase the ion beam-laser beam interaction by making the two beams nearly collinear over $250 \mathrm{~mm}$; (2) decelerate the precursor ion beam from $10 \mathrm{keV}$ to $100 \mathrm{eV}$ in the interaction region, thereby increasing ion density in the PID cell; (3) use an excimer laser of $400 \mathrm{~Hz}$ repetition rate. These changes should improve the fragmentation efficiency $10 \times 10 \times 4=400$-fold. Pulsing the $\mathrm{Cs}^{+}$ion beam [7] in synchronization with the laser beam to maximize utilization of the precursor ion generated from the sample should further increase the efficiency, theoretically to $100 \%$. The use of an excimer laser also permits the investigation of different wavelengths.

\section{Acknowledgment}

We would like to thank Questek Inc., Btllerica, Massachusetts, for a two-week loan of the excimer laser used in these experiments. This work was supported by grants RR00317 and ES04675 from the National Institutes of Health.

\section{References}

1. Bowers, W. D.; Delbert, S.-S.; Hunter, R. L.; McIver, R. T. J. Am. Chem. Soc. 1984, 106, 7288-7289.

2. Hunt, D. F.; Shabanowitz, J.; Yates, J. R. J. Chem. Soc., Chem. Commun. 1987, 8, 548-550.

3. Sato, K.; Asada, T.; Ishihara, M.; Kunihiro, F.; Kammei, Y.; Kubota, E.; Costello, C. E.; Martín, S. A.; Scuble, H. A.; Biemann, K. Anal. Chem. 1987, 59, 1652-1659.

4. Hill, J. A.; Martin, S. A.; Biller, J. E.; Biemann, K. Biomed. Environ. Mass Spectrom. 1988, 17, 147-151.

5. Hill, J. A.; Biller, J. E.; Martin, S. A.; Biemann, K.; Yoshidome, K.; Sato, K. Int. J. Mass Spectrom. Ion Processes 1989, 92, 211-230.

6. Martin, S. A.; Johnson, R. S.; Costello, C. E.; Biemann, K. In Analysis of Peptides and Proteins by Mass Spectrometry; McNeal, C. J., Ed.; Wiley: New York, 1988; pp 135-150.

7. Tecklenburg, R. E.; Castro, M. E.; Russell, D. H. Anal. Chem. 1989, 61, 153-159. 